area, Sri Lanka. A baseline survey was conducted from January to September 2007 and a follow-up survey was conducted from March to November 2010. Mortality data were obtained from next of kin and cause of death was verified from death certificates.

Results There were 49 deaths during 9186.46 person years of observations. Of the 49 deaths, 11 were due to myocardial infarctions, 5 were due to strokes, 5 were due to other ischaemic heart disease and the rest included 6 due to cancer and 2 due to train accidents. The increase in mortality in men occurs after 45 years and in females it is observed later on. Mortality among men was more than twice as much as females (RR 7.96 vs 3.17 per 1000 person years). All cause mortality was significantly higher in diabetics. Mortality was not associated with hypertension, dyslipidaemia, smoking, central obesity, obesity or physical activity.

Conclusions Diabetes Mellitus was significantly associated with all cause mortality. Other associations may have not been significant due to the small number of deaths.

\section{SP1-89 TENDENCY OF HORMONE REPLACEMENT THERAPY (HTR) USE IN A 18-YEAR FOLLOW-UP RETROSPECTIVE COHORT OF WOMEN IN THE VALENCIAN COMMUNITY (VC)}

doi:10.1136/jech.2011.142976n.66

\begin{abstract}
${ }^{1,2} \mathrm{R}$ Peiró-Pérez, ${ }^{1,3} \mathrm{D}$ Salas, ${ }^{1} \mathrm{G}$ Vallés, ${ }^{1,3} \mathrm{~J}$ Miranda, ${ }^{1,3} \mathrm{~J}$ Ibañez, ${ }^{1} \mathrm{~A}$ Molina-Barceló, 1,3E Pérez-Sanz.* ${ }^{1}$ Centro Superior de Investigación en Salud Pública (CSISP), Valencia, Spain; ${ }^{2}$ Ciber de Epidemiología y Salud Pública (CIBERESP), Madrid, Spain; ${ }^{3}$ Dirección General de Salud Pública, Valencian Community, Spain
\end{abstract}

Objective To described the tendency of Hormone Replacement Therapy (HTR) use in a 18-year follow-up retrospective cohort of women participating in the Valencian Community (VC), breast cancer screening program.

Methods Between 1992 and 2009, a retrospective cohort of participants in a population-based breast cancer-screening program in the VC was assessed. The study population was 683739 women, 45-69 years. Trends in current HTR use, and new and leaving users, by educational level (EL) and age group were analysed. A regression analysis by the joint point (JP) for the tendency was calculated.

Results From 1992 to 2009, the $11.73 \%(N=71827)$ of women was taken HRT, annual increases were found in the prevalence levels of HRT use to 2003 (13.5\%) and remains in 2009 (12.5\%). The peak by aged group was $20 \%$ in 2003 for the 55-59 group. The new users of HRT have increased until 1998, changing significantly, to decrease until 2009, as shows the JP regression analysis $(p<0.005)$. The university (EL) new user's peak was in 1999 (12.3\% vs 3.6\%) in the no-studies group, in 2002 (8.2\% vs $2.9 \%$ ), in 2009 (2.7\% vs $0.7 \%$ ) $(p<0.0001)$. The university leaving use peak was in 2004 (1.4\% vs $1.05 \%)$ in the no-studies, in $2009(0.4 \%$ vs $0.7 \%)$. There is a statistically significant difference in HTR users by EL, the regression analysis of the JP, shows that the trend is parallel increasing until the year 1998, and began a reduction until 2009.

Conclusions No too much impact had the WHI study in reduction of percentage of women taking HRT in our study.

\section{SP1-90 SOCI0-DEMOGRAPHIC AND GEOGRAPHIC DIFFERENCES IN HEALTHCARE RELATED OUTCOMES IN A COHORT OF DIABETES PATIENTS IN THE LAZIO REGION, ITALY}

doi:10.1136/jech.2011.142976n.67

U Kirchmayer, * V Belleudi, L Bauleo, N Agabiti, L Pinnarelli, D Fusco, M Arcà, M Davoli. Department of Epidemiology of the Regional Health Service, Lazio Region, Italy

Introduction The Italian health system guarantees care to all citizens. Previous research reported socio-demographic differences in access to evidence-based therapies, whereas studies evaluating differences in healthcare related outcomes are scarce.

Objectives Analysing socio-demographic and geographical differences in healthcare related outcomes in patients with diabetes mellitus (DM).

Methods Among all residents registered with the regional healthcare service in 2008, adult patients with DM were identified through a validated predictive model from the regional administrative health information systems (hospital discharges, drug claims, exemptions for DM). Healthcare related outcomes were hospitalisation for uncontrolled DM, short-term complications, long-term complications, and amputation of lower limb (excluding traumatic amputations). Age standardised rates were calculated by gender, geographic area and socio-economic position (SEP) of residence (only for the city of Rome, 5 levels), and RRs were calculated.

Results In 2008, 2569 cases of complications were registered among the 303.016 DM patients, with higher prevalence among males. Incidence rates varied between $0.8 / 1000$ for short-term and 4.2/1000 for long-term complications. Geographic variations were not statistically significant. Incidence of admissions for uncontrolled DM and short-term complications increased with decreasing socioeconomic position in both genders. A significant risk increase was observed among males with lowest respect to highest SEP for amputations ( $R R=5.88,95 \%$ CI 1.69 to 20.51) and for short-term complications ( $\mathrm{RR}=12.58,95 \% \mathrm{CI} 2.83$ to 55.91 ).

Conclusions In the Lazio region, complications of DM are more frequent among males. In Rome, the risk of complications is higher in DM patients belonging to lower SEP.

\section{SP1-91 NON-INVASIVE ASSESSMENT OF HEPATIC INJURY IN CHRONIC LIVER DISEASES: IMPLEMENTATION IN CLINICAL PRACTICE. A COMPARATIVE STUDY IN MANIPAL TEACHING HOSPITAL OF WESTERN NEPAL}

doi:10.1136/jech.2011.142976n.68

${ }^{1}$ A Mittal, ${ }^{1} \mathrm{~B}$ Sathian, ${ }^{*}{ }^{2} \mathrm{~A}$ Kumar, ${ }^{1} \mathrm{~N}$ Chandrasekharan. ${ }^{1}$ Manipal College of Medical Sciences, Pokhara, Nepal; ${ }^{2}$ College of Medicine \& JNM Hospital, West Bengal, India

Background Chronic hepatitis B and C together with alcoholic (ALD) and non-alcoholic fatty liver diseases (NAFLD) represent the major forms of progressive liver disease.

Objective To assess the extent of liver injury by estimation of biochemical parameters and its significance in clinical practice.

Materials and Methods It was a retrospective study carried out from the data maintained in the Department of Biochemistry of the Manipal Teaching Hospital, Pokhara, Nepal between 1 June 2009 and 31 July 2010. Descriptive statistics and testing of hypothesis were used for the analysis using SPSS 16 software.

Result Of the 653 subjects, $48.5 \%$ were controls, $19.6 \%$ ALD, Viral Hepatitis $18.4 \%$ and $13.5 \%$ had NAFLD. In cases of viral hepatitis, mean values of aspartate aminotransferase and alanine aminotransferase were markedly increased as compared to controls. However, in alcoholic patients, ALT level is usually elevated to a lesser degree than AST level. The mean value of AST was markedly increased in comparison to ALT leading to significantly higher AST/ ALT ratio 1.59 with CI (1.49 to 1.69) ( $p=0.001)$. In NAFLD patients, there was mild to moderate elevation in serum levels of AST, ALT or both. The mean value of the ratio of AST/ALT was $1.03 \pm 0.26$ but this ratio increases as fibrosis advances, leading to a loss of its diagnostic accuracy in patients with cirrhotic non-alcoholic fatty liver disease.

Conclusion Simple, cheap and reliable non-invasive biochemical markers should be used for early stage detection of liver diseases especially the countries having low socioeconomic status like Nepal. 